\title{
Assemblée générale 2013 de la SMF
}

C 'est dans la salle Robert Genot, à Saint-Mandé (94), que s'est tenue, le 7 mars dernier, l'assemblée générale 2013 de la SMFMétéo et Climat, en présence d'une quarantaine d'adhérents.

Lors de l'ouverture de la séance, le président Jean Jouzel a rendu hommage à André Lebeau décédé le 26 février, qui a présidé l'association de 1997 à 2011.

La secrétaire générale Nicole Papineau a ensuite présenté le rapport moral 2012, décrivant les activités de fonctionnement courant et de rayonnement scientifique de la SMF-Météo et Climat.

Elle a rappelé les soutiens essentiels dont l'association a bénéficié en 2012 de la part de Météo-France, du CNRSInsu, de l'Ademe, des partenaires de la journée scientifique et du Forum International de la Météo et du Climat. Elle a fait état d'un nouveau soutien obtenu de la part du CNES.

Concernant les moyens financiers, elle a souligné la situation préoccupante qui a conduit l'association à prendre des décisions difficiles afin de rééquilibrer le budget. Parmi ces mesures, l'association a dû avec regret se séparer de Nathalie Rauline qui travaillait au rayonnement de la section régionale Midi-Pyrénées. Nous la remercions pour le travail accompli à Toulouse depuis 2000.

Les mesures d'économie ont également entraîné la dénonciation de la convention de mise à disposition par Météo-France d'un de ses agents pour le poste de secrétaire de rédaction de La Météorologie. Cette convention prévoyait la prise en charge par la SMF-Météo et Climat de la moitié du salaire de cet agent. La SMF-Météo et Climat et la rédaction de $L a$ Météorologie remercient vivement
Marie-Josette Régis pour sa contribution à la revue pendant plusieurs années.

Autre point important souligné par la secrétaire générale dans son rapport moral : la mise en place d'une campagne d'adhésions et d'abonnements auprès des laboratoires de la communauté scientifique, des universités et prochainement de sociétés privées.

Nicole Papineau a ensuite énoncé les principales activités d'ordre scientifique que la SMF-Météo et Climat a organisé au cours de l'année écoulée. Parmi celles-ci, elle a mentionné le $9^{e}$ Forum International de la Météo et du Climat qui s'est déroulé en mars 2012 à Genève, au siège de l'Organisation météorologique mondiale, les prix André Prud'homme et Perrin de Brichambaut, ainsi que les activités de la section régionale Midi-Pyrénées (cycle de débats « Autour d'un micro avec Joël Collado ", $9^{\text {es }}$ rencontres régionales Météo-Jeunes...). Enfin, elle a cité la journée scientifique « La prévision météorologique et hydrologique au cœur de l'économie et de la société » qui s'est déroulée en novembre à l'École normale supérieure. La plupart de ces événements ont été - ou seront - relatés en détail dans les numéros de La Météorologie.

À l'issue de cette présentation, le président a demandé l'approbation de l'assemblée. Le rapport moral 2012 a été adopté à l'unanimité.
Nicole Papineau a ensuite pris le relais pour présenter le bilan financier de l'exercice 2012 et le budget prévisionnel 2013. Ces éléments ont été adoptés à l'unanimité par l'assemblée et la SMF-Météo et Climat les tient à la disposition de ceux qui souhaiteraient les obtenir.

Julien Duffau, du cabinet Euraudit n'ayant pu assister à l'assemblée générale, le trésorier Nicolas Bériot a ensuite lu le rapport du commissaire aux comptes sur l'exercice 2012. Ce rapport précise qu'il n'a pas été constaté d'anomalie significative, que les comptes ont été certifiés réguliers et sincères et qu'ils donnent une image fidèle de la situation financière de l'association à la fin de l'exercice. Le même constat a été fait par le vérificateur aux comptes Frédéric Avril.

Jean Jouzel a ensuite donné les résultats des votes des adhérents qui ont permis d'élire au Conseil trois nouveaux membres :

- Sylvie Joussaume, directrice de recherche au CNRS, affectée au LSCE/IPSL.

- Christophe Cassou, chargé de recherche au CNRS, affecté au Cerfacs.

- Dominique Marbouty, membre du Conseil général de l'environnement et du développement durable et président de l'European Meteorological Society.
Sylvie Joussaume

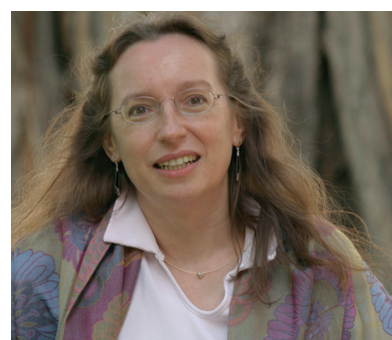

Christophe Cassou

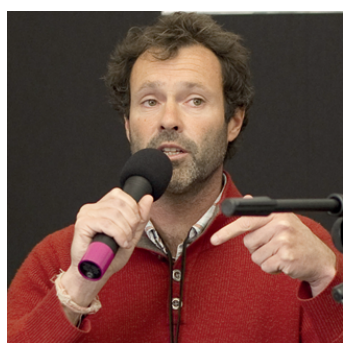

Dominique Marbouty

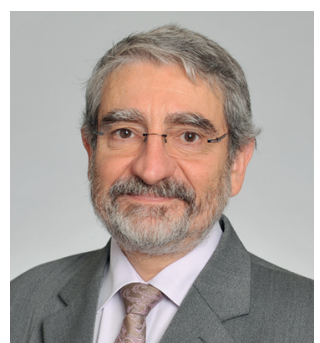


Ces nouveaux membres remplacent au Conseil Guy Lachaud, Daniel Martin et René Morin. Nous les remercions tous les trois pour leur contribution à la vie de l'association.

Les six autres membres du Conseil dont le mandat arrivait à terme ont été réélus : Guy Blanchet, Jean-Pierre Chalon, Joël Collado, Morgane Daudier, Laurent Garcelon et Matthias Gonzalez.

Le Conseil s'est ensuite brièvement réuni afin d'élire le Bureau.

Jean Jouzel a été reconduit dans sa fonction de président, Anne Guillaume et Stéphane Hallegatte en tant que vice-présidents. Nicole Papineau est reconduite dans sa fonction de secrétaire générale. Nicolas Bériot est réélu en tant que trésorier et Michel Petit en tant que conseiller. Deux nouveaux membres sont élus en tant que conseillers : Valérie Masson-Delmotte et Dominique Marbouty. La liste complète des membres du Conseil et du Bureau pour l'exercice 2013-2014 est donnée page 14 .

Jean Jouzel s'est ensuite adressé à l'assemblée afin de présenter les orientations futures de l'association, dans la ligne du plan stratégique adopté par le conseil d'administration en novembre 2010. L'association continue ses activités à destination des professionnels et

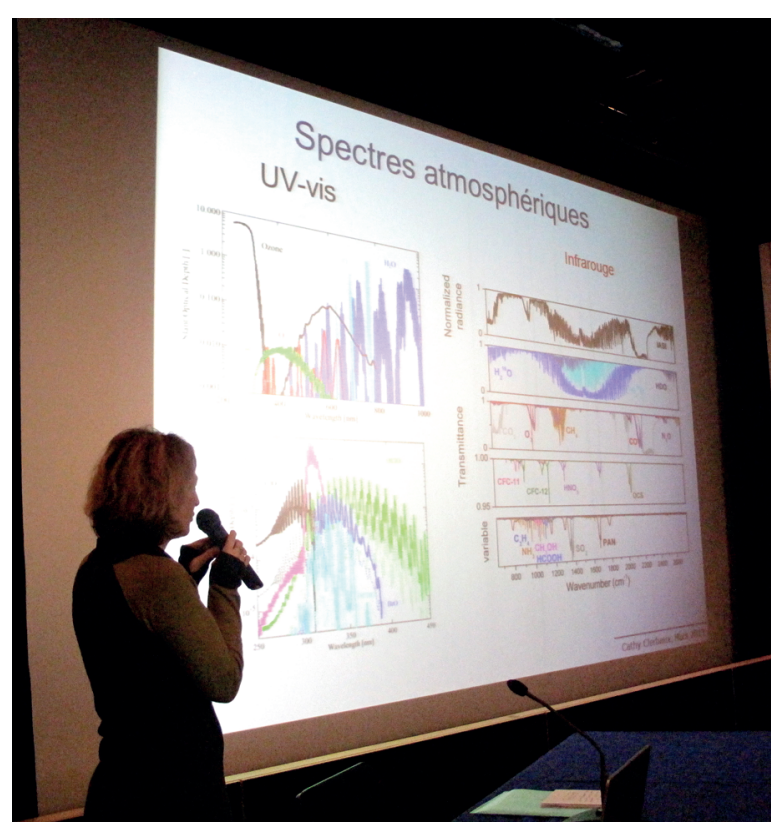

Cathy Clerbaux pendant son exposé sur les satellites. (C) Morgane Daudier. des amateurs et doit poursuivre la campagne d'adhésions et d'abonnement ainsi que la recherche de nouveaux partenaires.

Comme la tradition le veut, l'assemblée générale a été suivie d'une conférence donnée par Cathy Clerbaux, directeur de recherche CNRS au Latmos/IPSL (Laboratoire atmosphères, milieux, observations spatiales) sur le thème « Surveiller l'atmosphère par satellite : que peut-on voir et mesurer ? » Après avoir décrit ce qu'il est possible de " voir » avec les satellites actuels, Cathy Clerbaux a présenté les sondeurs les plus récents qui permettent de donner des alertes en temps réel quand des épisodes exceptionnels se produisent, par exemple en cas de pollution importante, de grands feux ou d'éruption volcanique.

L'assistance s'est ensuite retrouvée autour d'un cocktail de clôture dans une atmosphère sympathique et conviviale.

Morgane Daudier 


\section{Liste des membres du Conseil d'administration et du Bureau}

(exercice 2013-2014)

\begin{tabular}{|c|c|}
\hline $\begin{array}{l}\text { Président } \\
\text { Jean Jouzel }\end{array}$ & $\begin{array}{l}\text { Directeur de recherche au CEA. } \\
\text { Ancien directeur de l'Institut Pierre-Simon Laplace. } \\
\text { Membre du Groupe intergouvernemental d'experts sur l'évolution du climat (GIEC). }\end{array}$ \\
\hline $\begin{array}{l}\text { Vice-présidente } \\
\text { Anne Guillaume }\end{array}$ & Chargée de mission pour le calcul scientifique, université Pierre-et-Marie-Curie. \\
\hline $\begin{array}{l}\text { Vice-président } \\
\text { Stéphane Hallegatte }\end{array}$ & $\begin{array}{l}\text { Chercheur en économie de l'environnement et en science du climat (Météo-France/Cired). } \\
\text { Actuellement chargé de mission à la Banque mondiale. }\end{array}$ \\
\hline $\begin{array}{l}\text { Secrétaire générale } \\
\text { Nicole Papineau }\end{array}$ & Directrice adjointe, Institut Pierre-Simon Laplace. \\
\hline $\begin{array}{l}\text { Trésorier } \\
\text { Nicolas Beriot }\end{array}$ & Secrétaire général de l'observatoire national sur les effets du réchauffement climatique (ONERC). \\
\hline $\begin{array}{l}\text { Conseiller } \\
\text { Dominique Marbouty }\end{array}$ & $\begin{array}{l}\text { Ingénieur général des Ponts, des Eaux et des Forêts. } \\
\text { Membre du Conseil général de l'environnement et du développement durable (CGEDD). } \\
\text { Président de l'European Meteorological Society. }\end{array}$ \\
\hline $\begin{array}{l}\text { Conseillère } \\
\text { Valérie Masson-Delmotte }\end{array}$ & $\begin{array}{l}\text { Directrice de recherches au LSCE (Laboratoire des Sciences du Climat et de l'Environnement, } \\
\text { Institut Pierre-Simon Laplace). }\end{array}$ \\
\hline $\begin{array}{l}\text { Conseiller } \\
\text { Michel Petit }\end{array}$ & $\begin{array}{l}\text { Ingénieur général des Télécommunications. } \\
\text { Ancien directeur général adjoint pour la recherche à l'École polytechnique. }\end{array}$ \\
\hline
\end{tabular}

\begin{tabular}{|c|c|}
\hline Jean-Claude André & $\begin{array}{l}\text { Ingénieur général des Ponts et Chaussées. } \\
\text { Ancien directeur du Cerfacs. }\end{array}$ \\
\hline Guy Blanchet & Directeur honoraire du Centre de climatologie (université de Lyon I). \\
\hline Jean-Pierre Chalon & $\begin{array}{l}\text { Ingénieur général des Ponts, des Eaux et des Forêts. } \\
\text { Conseiller pour la communication scientifique à Météo-France. } \\
\text { Expert auprès de l'Organisation Météorologique Mondiale pour les questions de recherche } \\
\text { en modification artificielle du temps. }\end{array}$ \\
\hline Cathy Clerbaux & $\begin{array}{l}\text { Directeur de recherche CNRS au Latmos (Laboratoire Atmosphères, Milieux, Observations Spatiales). } \\
\text { Enseignante à l'université libre de Bruxelles. } \\
\text { Chercheur associée au NCAR (National Center for Atmospheric Research, États-Unis). }\end{array}$ \\
\hline Joël Collado & $\begin{array}{l}\text { Prévisionniste Météo-France } \\
\text { Chroniqueur météo sur France Info et intervenant spécialisé sur France Inter. } \\
\text { Président de la section régionale Midi-Pyrénées de Météo et Climat. }\end{array}$ \\
\hline Morgane Daudier & Administrateur Météo et Climat, chargée de la communication. \\
\hline Pierre de Félice & $\begin{array}{l}\text { Ancien professeur à l'université Paris XII (Créteil). } \\
\text { Laboratoire de météorologie dynamique. }\end{array}$ \\
\hline Laurent Garcelon & $\begin{array}{l}\text { Agent de maîtrise chez France Télécom Orange. } \\
\text { Correspondant et observateur bénévole pour Météo-France. } \\
\text { Administrateur de l'association Infoclimat. }\end{array}$ \\
\hline Matthias Gonzalez & $\begin{array}{l}\text { Informaticien chez Ligne Roset/Cinna. } \\
\text { Co-fondateur et trésorier de l'association Infoclimat. }\end{array}$ \\
\hline Sylvie Joussaume & $\begin{array}{l}\text { Directrice de recherches CNRS au LSCE (Laboratoire des Sciences du Climat et de I'Environnement, } \\
\text { Institut Pierre-Simon Laplace). } \\
\text { Directrice du GIS Climat-Environnement-Société. Membre du GIEC. }\end{array}$ \\
\hline Hervé Le Men & Ancien Ingénieur général des Ponts et Chaussées à l'IGN. \\
\hline Didier Renaut & $\begin{array}{l}\text { Ingénieur en chef des Ponts, des Eaux et des Forêts. } \\
\text { Ancien responsable de programme "Atmosphère météo et climat " } \\
\text { au Centre national d'études spatiales (Cnes). }\end{array}$ \\
\hline Michel Rochas & ieur général des Ponts, des Eaux et des Forêts. \\
\hline
\end{tabular}




\section{Forum international de la Météo et du Climat Un $10^{\circ}$ anniversaire couronné de succès}

Depuis 2004, I'association SMF-Météo et Climat donne rendez-vous au public mais aussi aux professionnels en co-organisant ce grand événement qui est devenu "un carrefour" annuel où une très grande diversité d'acteurs de la société civile se retrouve pour croiser des points de vue, exprimer des attentes, diffuser les connaissances, donner un élan aux services opérationnels et aux services naissants (océanographie, qualité de l'air, climatiques...).

Le Forum international de la Météo et du Climat est également devenu le lieu où les adultes de demain avec leurs enseignants viennent comprendre les enjeux d'un monde plus écologique et soucieux de l'avenir de notre Planète Terre.

\section{Jean Jouzel, président de la SMF-Météo et Climat}

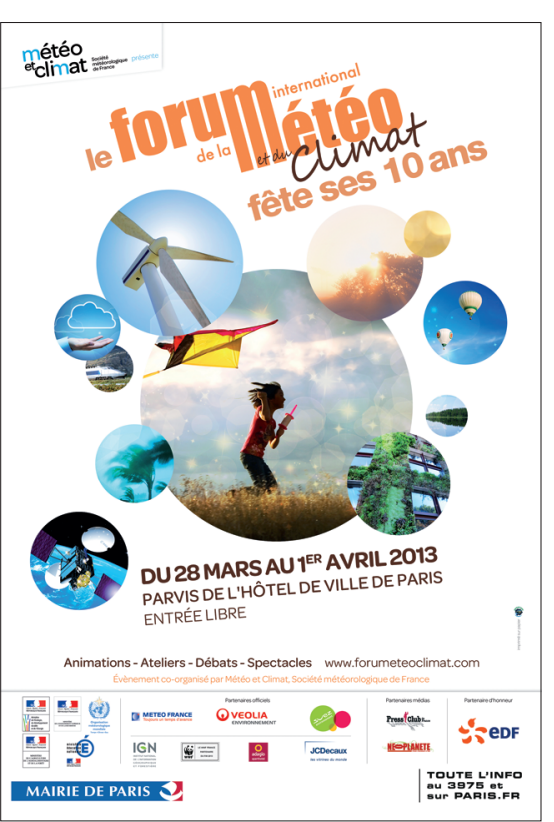

'est au cœur de Paris, sur le parvis de l'hôtel de ville récemment rebaptisé «Esplanade de la Libération », que le Forum a célébré ses 10 ans du 28 mars au $1^{\text {er }}$ avril 2013, sous le parrainage de la navigatrice Isabelle Autissier, présidente du WWF France.

Pour la première fois depuis sa création, le volet « professionnel » s'est tenu en amont du volet « grand public », du 21 au 23 mars.

\section{Le volet professionnel}

\section{Le colloque international}

Ayant pour thème « Agir face aux risques météo-climatiques : outils et enjeux économiques à l'horizon $2030 »$, le colloque international s'est tenu le 21 mars au Conseil économique, social et environnemental (Cese), siège du Palais d'Iéna. Après l'ouverture officielle par Isabelle Autissier et Jean Jouzel, la maîtresse de cérémonie Évelyne Dhéliat, chef du service météo de TF1 et de LCI, a passé le relais à Dominique Marbouty qui présidait la session 1 consacrée à l'état de l'art sur les prévisions météorologiques qui, d'ici une quinzaine d'années, permettront de mieux anticiper et donc de mieux gérer les risques météoclimatiques.

Un premier exposé a été présenté par Roberto Buizza, chef de la division prévisibilité au Centre européen pour les prévisions météorologiques à moyen terme, suivi d'un focus sur la prévision immédiate par Hans-Joachim Koppert, chef du service des prévisions au Deutscher Wetterdienst (service météorologique allemand). Après un éclairage sur la prévision climatique donné par Sylvie Joussaume, directeur de recherche au CNRS, Johannes Schmetz, chef de la division météorologie à Eumetsat, a concentré son exposé sur l'observation satellitaire.

L'assistance s'est ensuite retrouvée pour le déjeuner officiel soutenu par la Fédération française des sociétés d'assurances, sous la présidence de madame Delphine Batho, ministre de l'Écologie, entourée - entre autres - de Jean Jouzel, de Jean-Paul Delevoye, président du Cese et de François Jacq, présidentdirecteur général de Météo-France.

Les discussions de la session 2 pilotée par Stéphane Hallegatte se sont ensuite concentrées sur l'impact économique des risques météo-climatiques et sur les stratégies pour les réduire ou les gérer. Un premier exposé sur la perception et la mémoire des risques a été donné par l'historien du climat Emmanuel Garnier. Les aspects « risques et réassurance » ont été abordés par Peter Taylor de l'Université d'Oxford et Luc de Lignières, directeur de la gestion des risques IARD et opérationnels chez AXA. Robert Slomp, conseiller au

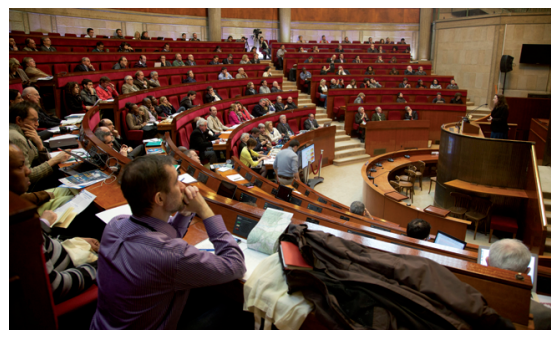

Hémicycle du Conseil économique, social et environnemental (Cese). (C) Anna Clopet

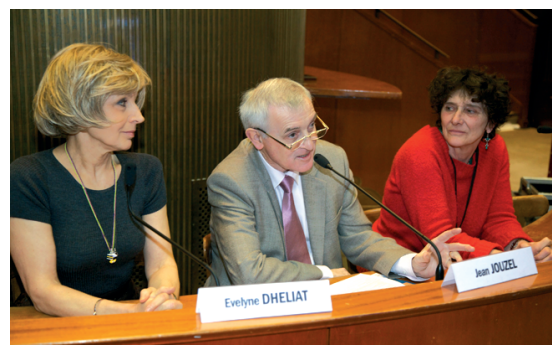

De gauche à droite : E. Dhéliat (TF1), J. Jouzel (climatologue et président de Météo et Climat) et I. Autissier (navigatrice). (C) Anna Clopet

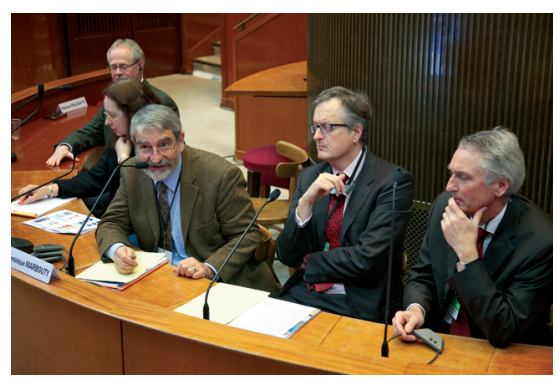

De gauche à droite : J. Schmetz (Eumetsat), S. Joussaume (CNRS), D. Marbouty (MEDDE/ CGEDD), R. Buizza (ECMWF) et H-J. Koppert (Deutscher Wetterdienst). (C) Anna Clopet

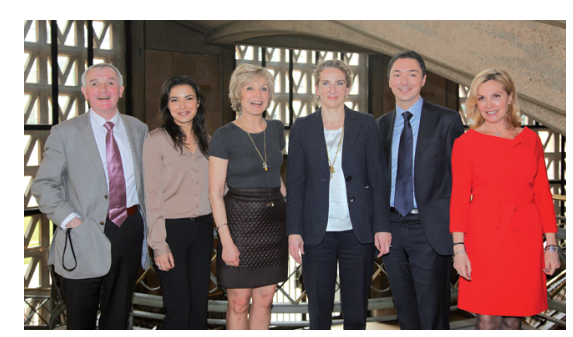

De gauche à droite : J. Jouzel, A. Baydemir (France 2), E. Dhéliat (TF1), D. Batho (ministre de l'Écologie), P. Verdier (France 2) et F. Amiach (France 3). (C) Anna Clopet 


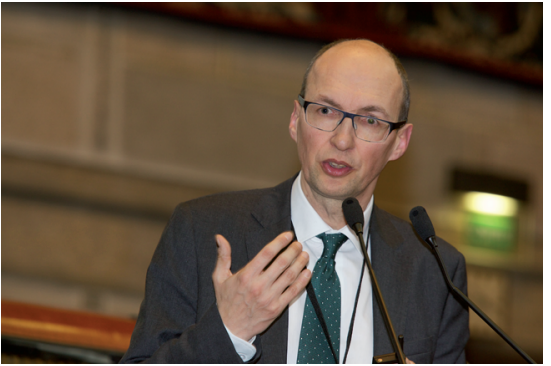

L. de Lignières (AXA). (C) Anna Clopet

service de la gestion de l'eau des PaysBas, s'est ensuite attaché à décrire le lien entre gouvernance des risques et réassurance. La session 2 s'est achevée avec l'exposé de Patrick Lagadec, directeur de recherche au Laboratoire d'économétrie de l'École Polytechnique sur le thème «Alerte et crise».

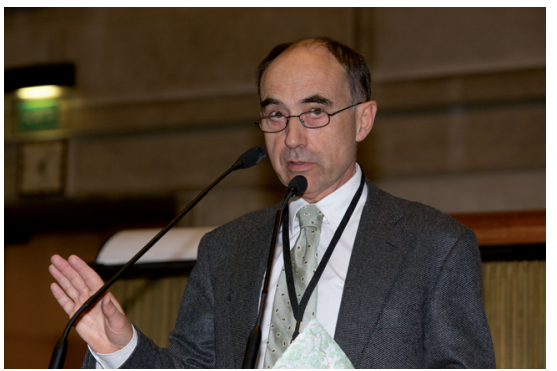

P. Lagadec (École Polytechnique). (C) Anna Clopet

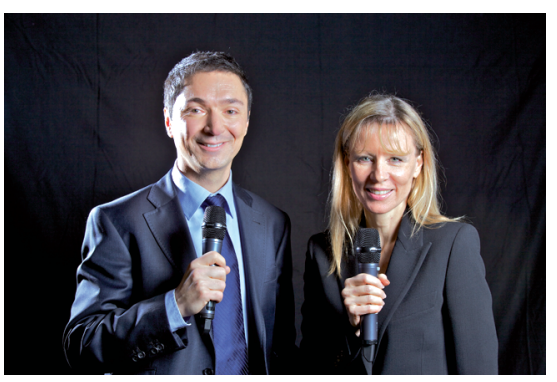

P. Verdier (France 2) et F. Klein (France 3). (C) Anna Clopet

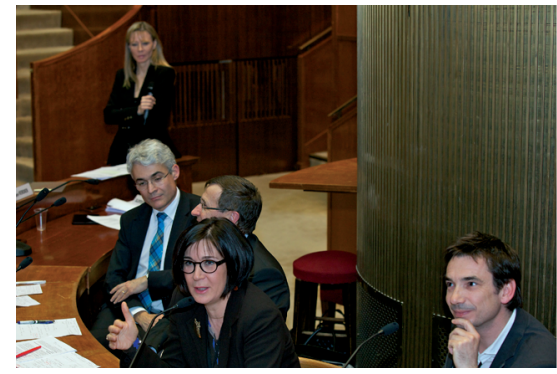

De gauche à droite : J.-P. Buisson (Dalkia), B. Guirkinger (Suez Environnement), V. David (Eiffage) et L.-S. Martin (EDF). (C) Anna Clopet

La table ronde de clôture sur le thème « La stratégie des entreprises face aux risques naturels » était animée par Florence Klein, chef du service météo de France 3 et Philippe Verdier, chef du service météo de France 2. Elle a réuni

\section{La formation pour les présentateurs météo des pays africains}

L'unité des services publics météorologiques (PWS) de l'Organisation météorologique mondiale a soutenu la formation pour les présentateurs météo des pays africains qui s'est déroulée le 22 mars 2013 au siège de Météo-France, à Saint-Mandé. Samuel Muchemi, agent scientifique de I'Unité PWS, en tant que représentant de I'OMM, a participé à la formation.

Le programme de la PWS a également financé partiellement la venue de certains experts, en complément de l'assistance généreuse du programme de l'OMM pour les pays les moins avancés (PMA). Les présentateurs météo du Burkina Faso, de Djibouti, du Malawi, du Mozambique, du Niger, du Swaziland et de la République Unie de Tanzanie ont bénéficié de cette formation qui était animée par Patrick de

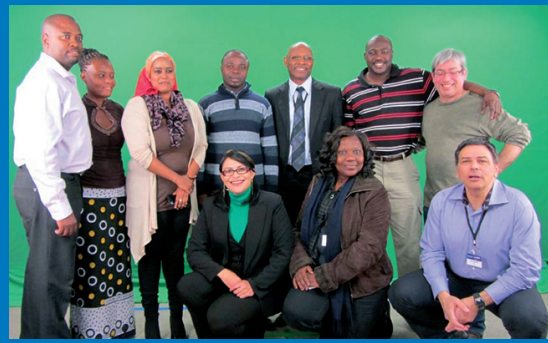

Les participants à la formation des pays africains 2013. (C) Louis Saubat-Lalanne

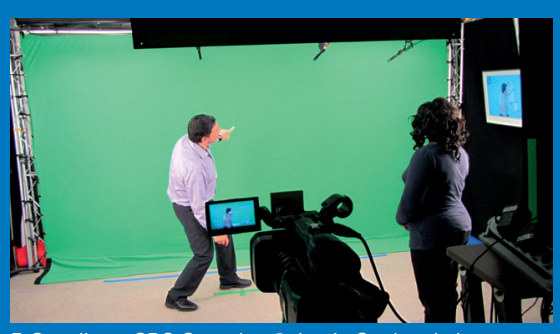

F. Cavallaro, CBC Canada. (C) Louis Saubat-Lalanne

dans le studio professionnel de l'unité média de Météo-France, mettant ainsi en œuvre leurs compétences nouvellement acquises. Il est important de souligner que cet événement annuel de la SMFMétéo et Climat donne l'occasion aux présentateurs météo du continent africain d'améliorer les messages météorologiques qu'ils délivrent aux téléspectateurs dans leurs pays respectifs et qu'il apparaît essentiel de poursuivre cette organisation dans le futur.

Samuel Muchem agent scientifique de l'Organisation météorologique mondiale

En haut, de gauche à droite : E. Tumaini (TBC1) MS. Lunda (MBC), P. Sihlongyane (SwaziTV) CJ. Sendela (MTV)

En bas, de gauche à droite : S. Bayala (BTV), II. Farah (DTV) et A. Anderson (NMS)

(c) Anna Clopet Bellefeuille, présentateur canadien de Météomédia et Franco Cavallaro de CBS Television et Radio, Montréal, Canada. La formation, proposée en français et en anglais, a été très stimulante, motivante et informative pour tous les participants qui ont pu apprendre à améliorer leurs compétences de présentation des bulletins météorologiques. Elle a en particulier mis l'accent sur la clarté de la voix, la position et le langage corporel et sur comment préparer au mieux le bulletin afin de transmettre un message essentiel aux téléspectateurs. Le programme comprenait une évaluation ainsi que des commentaires sur les bulletins préenregistrés, ce qui a permis aux participants et aux formateurs d'échanger leurs idées sur comment chacun pourrait améliorer sa présentation dans le futur. La partie pratique a permis aux participants d'enregistrer de vrais bulletins

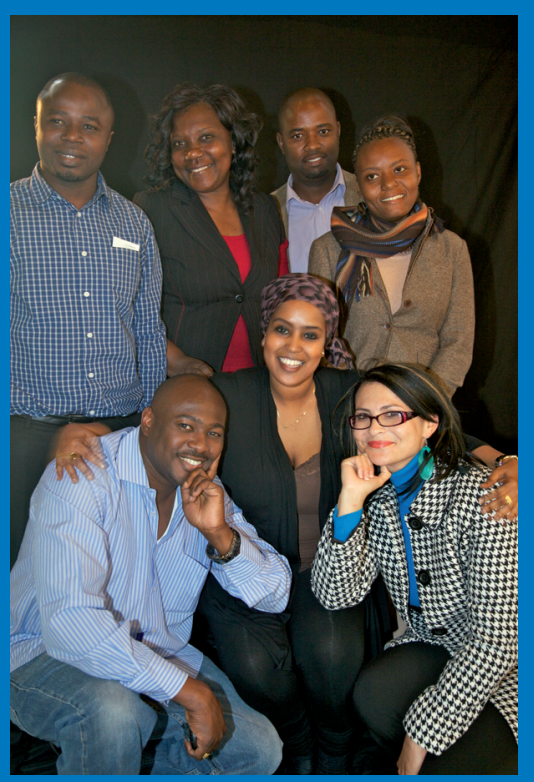

Bernard Guirkinger, directeur général adjoint de Suez Environnement, Laurent-Stéphane Martin, chef du groupe météorologie appliquée et environnement atmosphérique à la $\mathrm{R} \& \mathrm{D}$ d'EDF, Valérie David, directrice du développement durable d'Eiffage et Jean-Philippe Buisson, directeur régional Île-de-France de Dalkia. Lors des interviews préparatoires, Philippe Verdier s'est nourri des retours d'expériences des participants. A son sens, ces 
histoires sont plus captivantes qu'un discours sur le développement durable, moins vivant pour le public averti du forum. Ce sont des expériences que Florence Klein et lui ont subtilement mises en parallèle pour aborder la problématique des risques naturels.

\section{Le Media-Workshop}

Près de 50 présentateurs météo venus de plus de trente pays ont assisté aux ateliers média qui leur étaient réservés. Durant la matinée du 22 mars, alors que quelques participants suivaient une formation spécifique pour les pays africains (voir encadré), une quarantaine était accueillie à La Défense, au ministère de l'Écologie et du Développement durable et de l'Énergie, par Laurent Michel, directeur général de l'énergie et du climat. Une première session était consacrée aux actions et projets des présentateurs météo en matière de sensibilisation du public. À travers le documentaire « Survivre dans la tempête », Jill Peeters, météorologue sur la chaîne belge VTM, a relaté sa rencontre en 2012 avec les meilleurs chasseurs d'orages aux États-Unis. L'Américain Jay Trobec (Kelo TV) a pris le relais pour présenter les images d'une tempête dans le centre nordaméricain. Humberto Delgado Rosa, directeur général des technologies à bas

" Pour un présentateur météo, il n’y a rien de plus enrichissant que de participer au Forum international de la Météo et du Climat. Visionner les bulletins météo d'autres pays du monde nous permet d'améliorer notre propre présentation et aussi de nous tenir au fait des nouvelles technologies. Les idées fusent dans les ateliers et discussions, et tous les présentateurs se sont accordés pour essayer de donner plus de visibilité au FIM dans leurs pays respectifs, à travers leur média. Cet événement est crucial à l'évolution du métier de présentateur météo. »

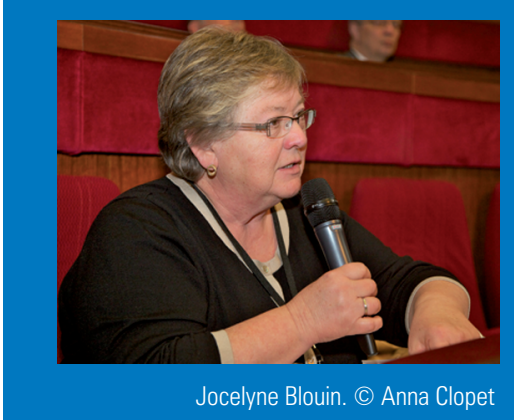

Jocelyne Blouin météorologue, ancienne présentatrice météo sur Radio Canada. carbone et de l'intégralité de l'adaptation à la direction générale climat de la Commission européenne a ensuite présenté un exposé sur le thème « Informer l'opinion publique du changement climatique : comment construire un monde que nous aimons, avec un climat appréciable ? \La matinée s'est achevée par une présentation d'Isabelle Derville, chef du bureau de la qualité de l'air au ministère de l'Écologie sur le thème de l'information sur la qualité de l'air.

Les sessions de l'après-midi ont proposé une séance de projection des bulletins météorologiques du monde entier, suivie des exposés de la présentatrice brésilienne Flavia Freire (TV Globo) et du Danois Jesper Theilgaard (DR).

À l'issue de la journée, le réalisateur belge Nic Balthazar, qui avait fait le déplacement à Paris, a présenté à l'ensemble des présentateurs l'action « Sing for the Climate » (chantez pour le climat) dont il est l'initiateur. Cette mobilisation symbolique contre le réchauffement climatique sera proposée dans le cadre de l'édition 2014 du Forum (http://www.singfortheclimate.com).

\section{Le volet grand public}

Pendant 5 jours, du 28 mars au $1^{\text {er }}$ avril, plus de 8900 visiteurs dont 730 scolaires ont été accueillis sur le parvis de l'hôtel de ville de Paris.

Le FIM, dont l'un des objectifs est de mieux informer et de sensibiliser le public aux enjeux du climat, a proposé des animations pédagogiques et des ateliers de vulgarisation scientifiques articulés autour de cinq thématiques : Météo-Climat, Eau, Environnement, Energies et Espace.

Dans le secteur Météo-Climat, MétéoFrance avait créé une animation autour des différents types de nuages, comment les reconnaître et comment se forment les tempêtes. Une expérience pratique permettait aussi de construire son propre thermomètre.

Le CEA, représenté par le Laboratoire des sciences du climat et de l'environnement, a démontré, à travers deux expériences, que l'océan est un acteur majeur du climat tandis que l'Institut national des sciences de l'univers (Insu) du CNRS a expliqué les raisons du changement climatique tout en rappelant le lien entre la température et la circulation océanique.
Pour sa première participation au Forum, l'association IFFO-RME (Institut français des formateurs risques majeurs et protection de l'environnement) a décrit le système de crues et d'inondations à travers des expériences ludiques et interactives.

La SMF-Météo et Climat a proposé aux jeunes visiteurs de créer un anémomètre afin de mesurer la vitesse ou la pression du vent.

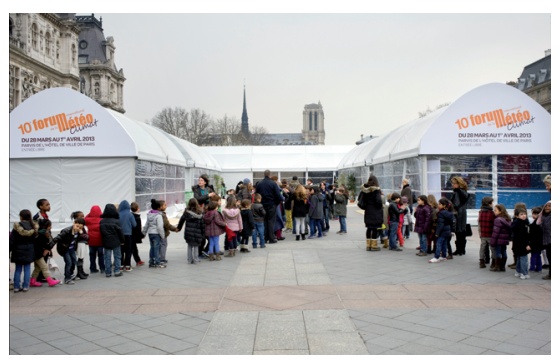

Vue générale du parvis de l'hôtel de ville de Paris. (C) Xavier Popy

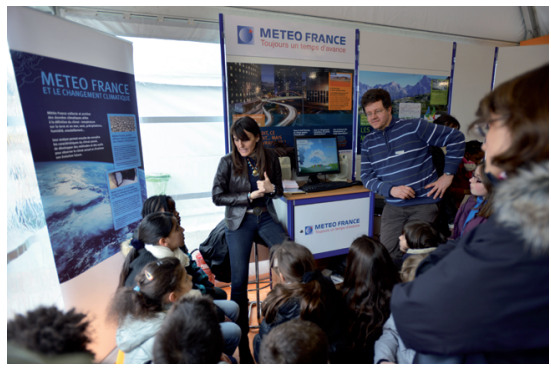

Animation « Comment élabore-t-on une prévision météorologique ? » par Météo-France. (C Pascal Taburet

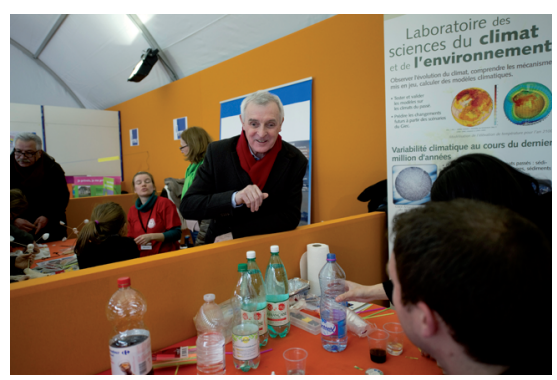

J. Jouzel sur l'espace du CEA/LSCE. (C) Xavier Popy

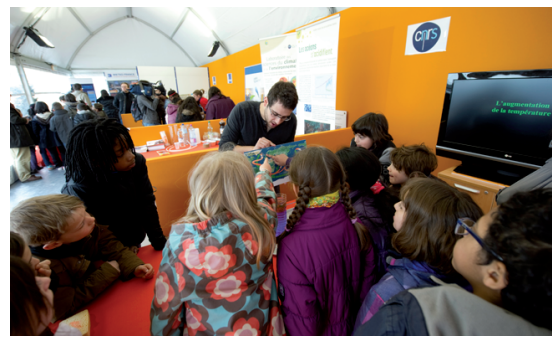

Animation "Quel climat pour demain ? " par le CNRS Insu. (C) Xavier Popy

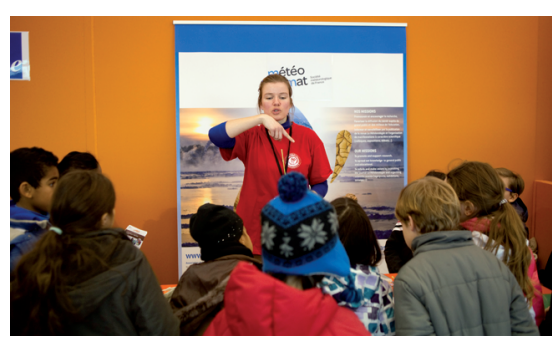

Animation « Réalise ton propre anémomètre » par l'association Météo \& Climat. (C) Xavier Popy 
Les inconditionnels des studios météo télé et radio ont pu s'exercer à la présentation d'un bulletin météo entraînés par des présentateurs du PAF, tels Sandra Larue de BFM TV ou Géraldine de Mori de RMC.

Dans le secteur Environnement, l'IGN, Institut national de l'information géographique et forestière, a sensibilisé les visiteurs à l'importance et à l'utilité de l'information géographique.

La SNCF a démontré qu'utiliser les transports en commun réduisait l'impact de l'homme sur l'environnement et faisait découvrir le développement de nouvelles énergies.

La fondation WWF France a donné l'occasion aux jeunes "pandas » de découvrir par quels moyens l'homme pouvait sauvegarder la biodiversité forestière de la planète.

L'association Objectif Sciences International, accueillie pour la première fois au Forum, a mis en scène une glace « $\mathrm{Mr}$ Freeze », bien connue et appréciée des enfants, afin d'expliquer comment retarder au maximum la fonte des glaces. Une dégustation était prévue à l'issue de l'expérience pour les petits gourmands.

Enfin, la Direction de la propreté et de l'eau de la Mairie de Paris a proposé une animation autour des enjeux du compostage, montrant comment composter et utiliser le compost.

Seul organisme présent dans le secteur Énergies, la SMF-Météo et Climat a expliqué le principe de l'énergie solaire et de l'albédo afin de montrer comment ce dernier intervient dans le système d'économie d'énergie.

Dans le secteur eau, le SIAAP (Syndicat interdépartemental pour l'assainissement de l'agglomération parisienne) a permis de suivre le cheminement de l'eau et de découvrir son passage dans les stations d'épuration méconnues du grand public.

Enfin, dans le secteur espace, l'ESA (agence spatiale européenne) a proposé un quizz interactif sur le rôle des satellites météorologiques aux côtés de l'association Planète Sciences qui a fait découvrir plusieurs types de risques météorologiques, à travers l'analyse d'images prises par Envisat.

Afin de marquer son anniversaire, le Forum a pu proposer un programme d'animations spéciales au cours du

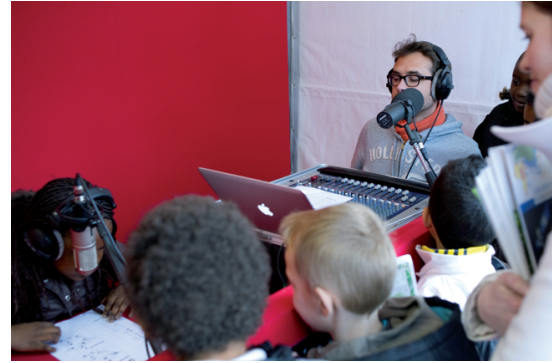

Animation radio avec R. Féokio. (C) Xavier Popy

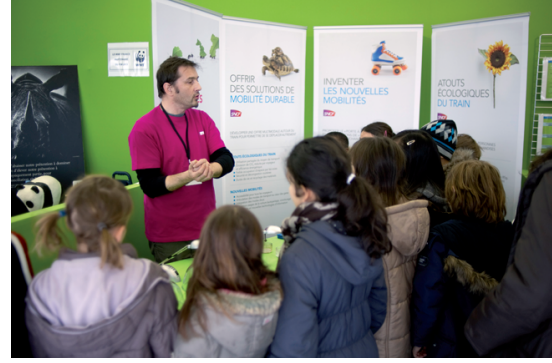

Animation « Les transports écologiques » par la SNCF. (C) Xavier Popy

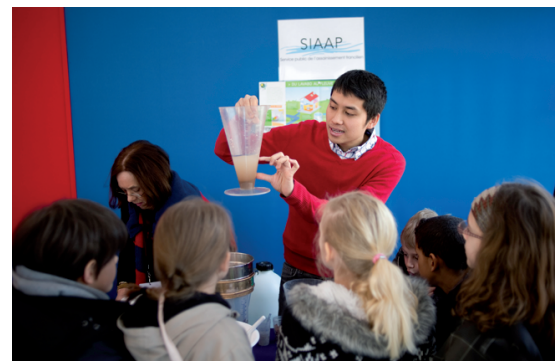

Animation " Lave ton eau » par le Syndicat Interdépartemental pour l'Assainissement de l'Agglomération Parisienne (SIAPP). C Xavier Popy

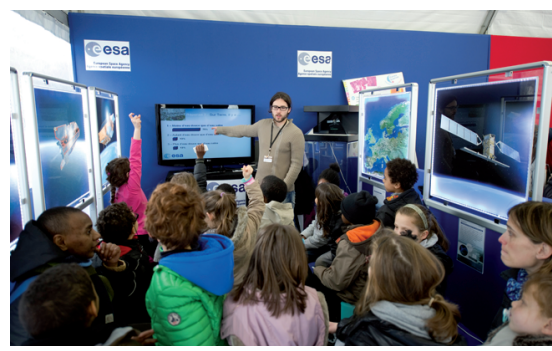

Animation " La Terre vue de I'Espace " par l'Agence Spatiale Européenne. (C) Xavier Popy

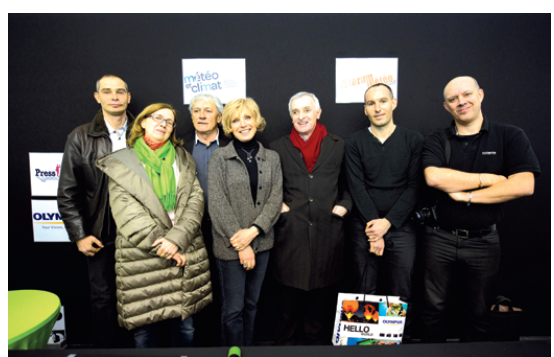

Remise des prix du concours photo « Les phénomènes météorologiques extrêmes "

De gauche à droite: $A$. Cabanié ( $2^{\circledR}$ prix), A. Guillaume (Météo \& Climat/UPMC), J. Collado (Météo-France), E. Dhéliat (TF1), J. Jouzel (Météo \& Climat), C. Leroy (1 ${ }^{\text {er }}$ prix) et T. Bourque (Olympus)

Retrouvez les photos sur www.forumeteoclimat.com week-end de Pâques. Le public a tout d'abord été convié à une rencontredébat avec Évelyne Dhéliat (TF1) et Joël Collado (Météo-France) sur le thème des coulisses du bulletin météo.

Pour la première fois, le Forum a organisé un concours photos, sur le thème des «phénomènes météorologiques extrêmes ». Parmi la centaine de photos reçues, 10 ont été sélectionnées par le jury afin d'être exposées sur le parvis pendant le Forum (voir page 22).

Les auteurs des trois meilleures photos ont été récompensés par un appareilphoto OMD d'Olympus, les ouvrages Earth et Power ainsi que des abonnements au magazine Réponses Photo. La remise des prix, animée par le journaliste David Kolski (Evasion), s'est déroulée en présence de Thierry Bourque d'Olympus, partenaire du concours photo, de Jean Jouzel, président de la SMF-Météo et Climat, d'Anne Guillaume, vice-présidente, d'Évelyne Dhéliat (TF1) et de Joël Collado (prévisionniste de MétéoFrance pour Radio France).

Deux questions à Alain Mazeau (climatologue et chargé de communication au Laboratoire des sciences du climat et de l'environnement)

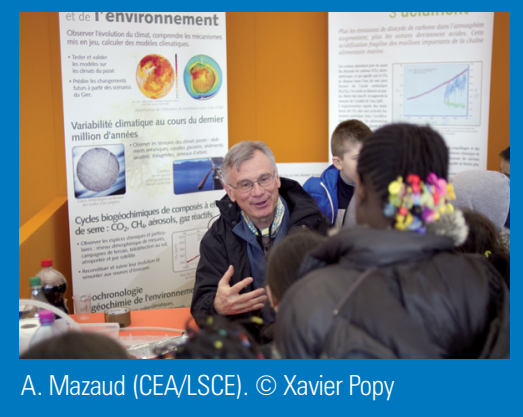

1) Comment adaptez-vous votre discours quand vous accueillez tantôt un très jeune public, tantôt un public plus averti ?

Pour les jeunes, nous proposons des petites expériences concrètes et utilisons un langage simple avec des analogies " comme si... » et pas de graphiques...

Pour un public plus averti, on peut entrer davantage dans les détails, montrer des graphiques et donner quelques chiffres (pas trop quand même).

2) Quel public était le plus captif ? Les élèves du primaire, ceux du secondaire ou les adultes?

Les élèves du primaire. Ceci ne veut pas dire que les autres ne l'étaient pas... Pour les adultes, les personnes accompagnantes (instituteurs, parents... étaient tout aussi intéressées que les enfants qu'elles encadraient. 


\section{Concours pour les écoles, les collèges}
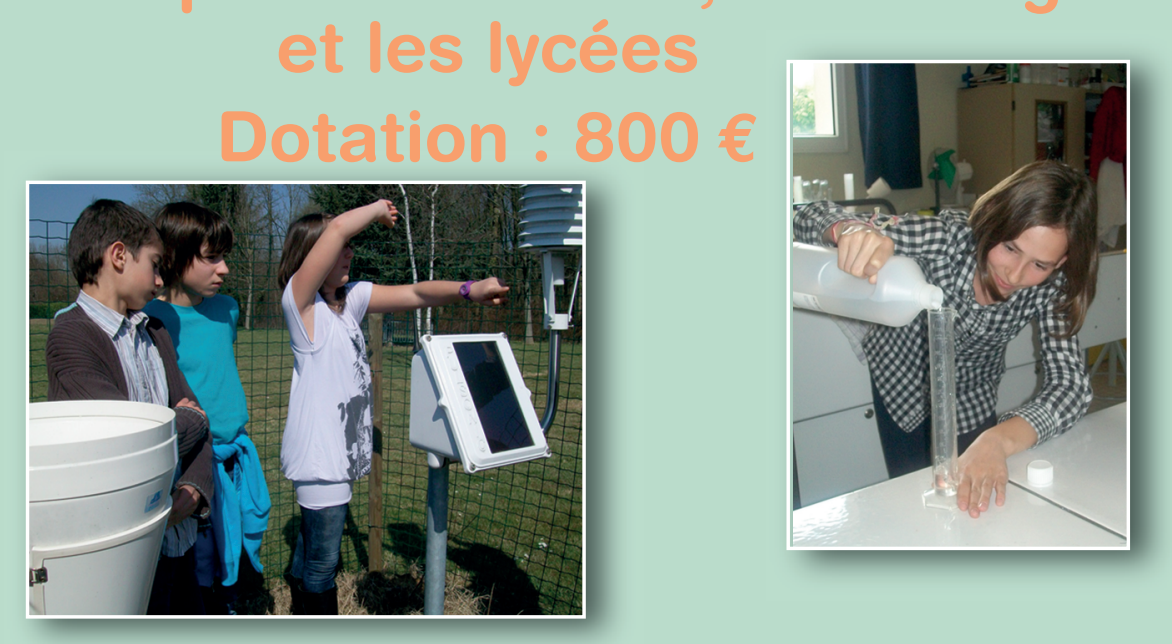

Ce prix a été créé par la SMF-Météo et Climat, Société météorologique de France, pour honorer la mémoire de Christian Perrin de Brichambaut, s'adresse aux écoles primaires, collèges et lycées qui réalisent un projet sur le thème de la météorologie ou du climat au cours de l'année scolaire 2013-2014.

Les projets sont évalués par un jury composé de météorologistes professionnels et d'enseignants.

$1^{\text {er }}$ prix : $800 €$ et présentation du projet dans La Météorologie, Revue de l'atmosphère et du climat.

Prix suivants : outils pédagogiques (livres, posters, CD, DVD...)

\section{Comment concourir au prix 2014 ?}

Envoyez votre dossier qui contient :

1 - une lettre de candidature avec les coordonnées de l'établissement et du responsable du projet (son $\mathrm{n}^{\circ}$ de téléphone et son adresse électronique), le nombre d'élèves impliqués, le niveau de la classe ;

2 - le descriptif du projet (une page format A4) ;

3 - tous les éléments permettant au jury d'apprécier le projet (dossier de présentation, illustrations, CD-ROM, DVD, ...).

\section{Où envoyer le dossier ?}

\section{SMF-Météo et Climat - Prix Perrin de Brichambaut - 73, avenue de Paris 94165 Saint-Mandé Cedex}

Date limite : les candidatures sont à envoyer avant le 31 mai 2014

\section{Exemples de projets}

Lâchers de ballons-sondes, observations et mesures en météorologie, création d'un site Internet, fabrication d'instruments...

\section{Attribution du prix}

Le prix sera remis dans l'établissement avant la fin de l'année scolaire 2013-2014.

Organisé par

météo

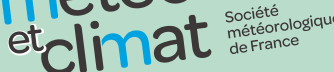

Avec le soutien de

METEO FRANCE

Toujours un temps d'avance

Informations complémentaires 0177947364 - morgane.daudier@meteoetclimat.fr www.meteoetclimat.fr 
Le dimanche de Pâques, alors que les plus gourmands se sont rassemblés autour d'une animation " chocolat», d'autres ont participé à une séance de dédicace de Bernard Thomasson, journaliste sur France Info pour son ouvrage Guide de voyage météo, coécrit avec le présentateur météo de TF1, Louis Bodin.

Enfin, le dernier jour, parents et enfants ont assisté à « Chaud devant », une représentation théâtrale donnée par la compagnie Pile Poil sur le thème du réchauffement climatique, traité de manière humoristique.

Les présentations des intervenants du colloque international et de la formation pour les enseignants sont téléchargeables sur le site :

\section{www.forumeteoclimat.com}

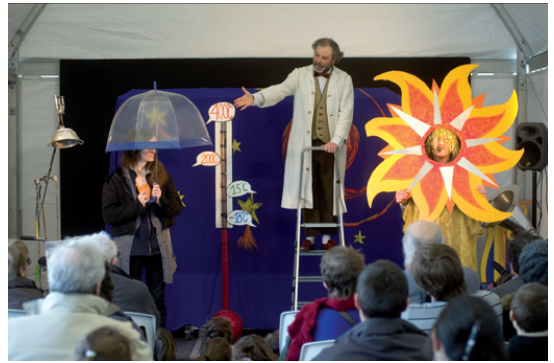

Spectacle « Chaud Devant » par la Compagnie Pile Poil. (C) Xavier Popy

\section{La formation pour les enseignants}

Les enseignants du primaire et du secondaire jouent un rôle déterminant dans la transmission des savoirs. La météorologie et le climat font partie du quotidien de chacun et les programmes scolaires français intègrent, de manière relativement morcelée, une éducation à la météorologie et au climat, répartie entre histoire-géographie, physique et sciences de la vie et de la terre.

Dans un contexte de progrès scientifiques, dans les observations, la compréhension des variations passées, présentes, et la modélisation qui permet l'anticipation des risques, il est indispensable de partager ces connaissances avec les enseignants.
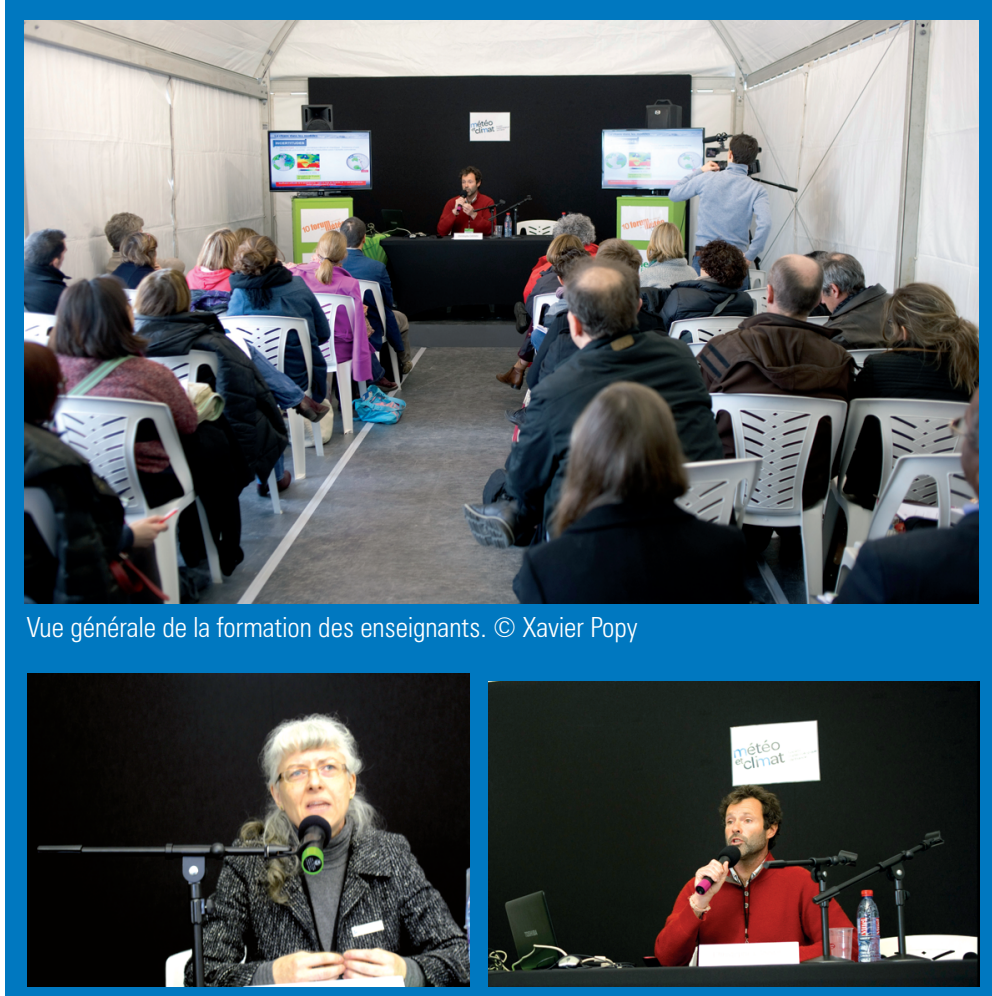

C. Delage, Météo-France.

(C) Morgane Daudier

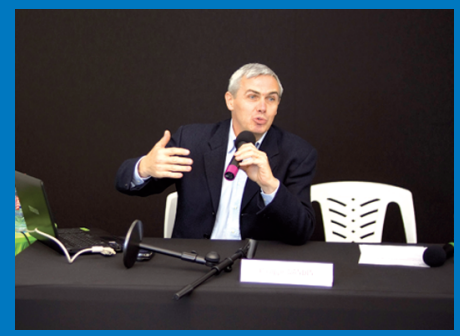

P. Dandin, directeur de la climatologie de Météo-France.

(C) Xavier Popy

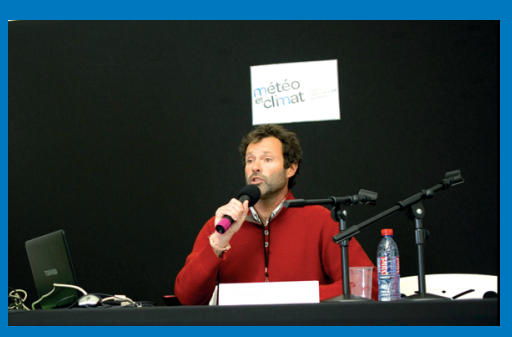

C. Cassou, CNRS/Centre européen de recherche et de formation avancée en calcul scientifique. (C) Xavier Popy

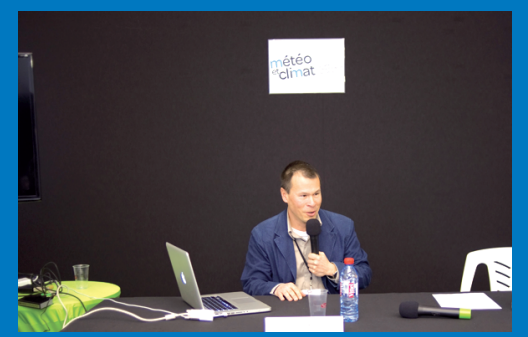

P. Yiou, Laboratoire des sciences du climat et de I'environnement, Institut Pierre Simon Laplace. (C) Xavier Popy Organisée avec le soutien de Schneider Electric, cette formation, qui s'est déroulée le 28 mars sur le parvis de I'hôtel de ville de Paris, a accueilli une quarantaine de participants dont 31 enseignants autour de la thématique des événements météorologiques extrêmes. Le programme avait été mis en place par Nicole Papineau, secrétaire générale de la SMF-Météo et Climat et directrice adjointe à l'IPSL et moi-même.

Catherine Delage, chargée de mission à la Direction des missions institutionnelles de Météo-France, a décrypté le dessous des cartes de vigilance et présenté les ressources pédagogiques proposées par Météo-France. L'exposé de Christophe Cassou du Cerfacs s'est focalisé sur la prévisibilité, des échelles de temps de la météorologie (jusqu'à deux semaines) à celles du climat (de la décennie au siècle), en expliquant les méthodes mises en œuvre et les incertitudes associées aux prévisions et projections, et en s'appuyant sur l'actualité météorologique d'un printemps particulièrement frais. Les efforts en cours pour exhumer les informations météorologiques anciennes des archives météorologiques nationales, menés conjointement par les Archives nationales et Météo-France, ont été présentés par Philippe Dandin, directeur de la climatologie à Météo-France. Cette présentation a stimulé des réflexions sur des regards croisés entre disciplines (histoire et météorologie, par exemple). Enfin, Pascal Yiou, du Laboratoire des sciences du climat et de l'environnement, a précisé les méthodes mises en œuvre pour comprendre les relations entre événements extrêmes (canicules, vagues de froid, tempêtes) et changement climatique. Les participants ont exprimé un vif intérêt pour les méthodes, résultats et ressources pédagogiques utilisables en classe.

V. Masson-Delmotte
CEA-CNRS-UVSQ/IPSL

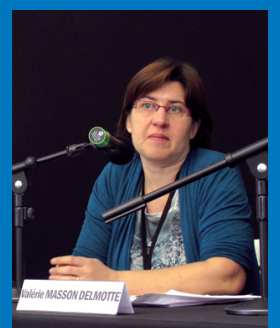

(c) Morgane Daudier 


\section{Remerciements}

Monsieur Bertrand Delanoë, maire de Paris.

Monsieur Jean-Paul Delevoye, président du Conseil économique, social et environnemental.

Isabelle Autissier, marraine d'honneur.

SOUS LE PATRONAGE DE

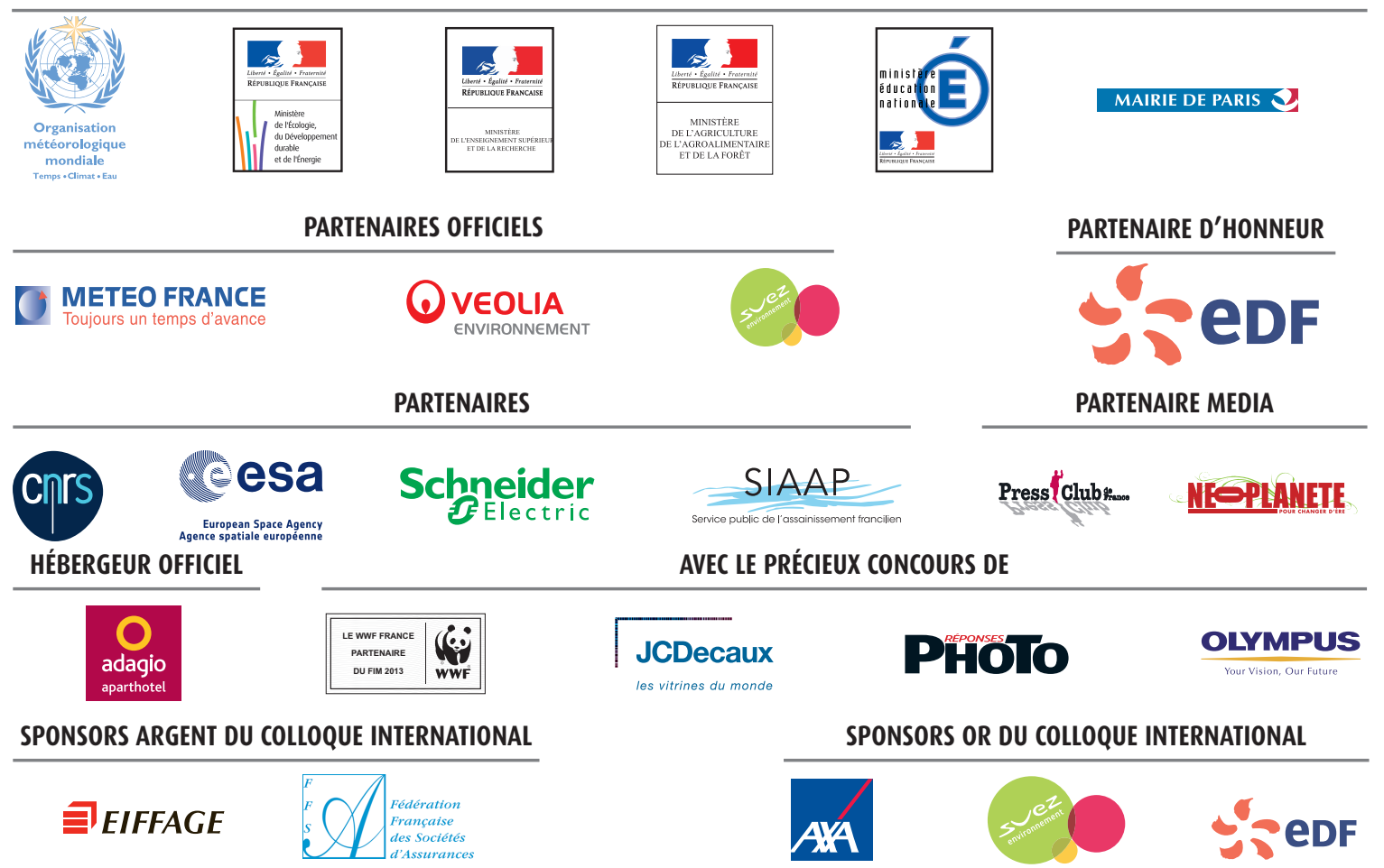

CEA, IGN, SNCF, Commission européenne/Direction générale Climat, contributeurs du Forum.

BNP Paribas, BRGM, CNES, Crédit Agricole Cheuvreux et Région île-de-France, sponsors Bronze du colloque international.

CEA/LSCE, Compagnie Nationale du Rhône, Essilor, ministère de la Culture et de la Communication / Délégation générale à la langue française et aux langues de France, contributeurs du colloque international.

Thierry Bourque (Olympus), Anne Guillaume (SMF-Météo et Climat), Isabelle Bourdet (Press Club de France), Michel Hontarrède (Météo-France), Nathalie Rihouet (France 2), Sylvie Hugues (Réponses Photo), membres du jury du concours photos.

Roberto Buizza (ECMWF), Hans-Joachim Koppert (DWD), Sylvie Joussaume (GIS Climat), Johannes Schmetz (Eumetsat), Emmanuel Garnier (Université de Caen/Université de Cambridge, Institut universitaire de France), Peter Taylor (Université d'Oxford), Luc de Lignières (AXA), Robert Slomp (Rijkswaterstaat Waterdienst), Patrick Lagadec (École Polytechnique), Bernard Guirkinger (Suez Environnement), Laurent-Stéphane Martin (EDF), Valérie David (Eiffage), Jean-Philippe Buisson (Dalkia) intervenants du colloque international du 21 mars.

Évelyne Dhéliat (TF1 et LCI), Florence Klein (France 3) et Philippe Verdier (France 2) pour avoir animé le colloque international du 21 mars.

Stéphane Hallegatte et Dominique Marbouty ainsi que les membres du comité scientifique pour la conception du programme et la présidence des sessions du colloque.

Samuel Muchemi (OMM), Patrick de Bellefeuille (MeteoMedia), Franco Cavallaro (CBS) intervenants de la formation pour les pays africains du 22 mars.

Laurent Michel (MEDDE/DGEC), Isabelle Derville (MEDDE/DGEC), Humberto Delgado Rosa (Commission européenne / DG Climat), Jill Peeters (VTM, Belgique), Nic Balthazar, Jay Trobec (Kelo TV, États-Unis), Flavia Freire (TV Globo, Brésil), Jesper Theilgaard (DR, Danemark) et Jocelyne Blouin intervenants du Media-Workshop du 22 mars.

Valérie Masson-Delmotte (LSCE/IPSL), Nicole Papineau (ISPL et SMF-Météo et Climat), Catherine Delage (Météo-France), Christophe Cassou (CNRS/Cerfacs), Philippe Dandin (Météo-France) et Pascal Yiou (LSCE/IPSL) intervenants de la formation des enseignants du 28 mars. Les chefs-restaurateurs, Jean-Jacques Noguier (La Ferme de I'Hospital à Bossey), Jean-Christophe Lebascle (La Manufacture à Issy-les-Moulineaux), Yvan Zaplatilek (Yvan à Tunis) et la brigade du restaurant La Manufacture pour leur accueil chaleureux à l'occasion du dîner de gala.

David Kolski (Radio Evasion) pour l'animation du studio météo télé.

Romann Feokio pour l'animation du studio météo radio.

Ana, Ana, Baptiste, Bojana, Chantal, Christian, Damien, Édouard, Isabelle, Jean, Jean-Philippe, Jérémy, Julie, Linda, Louis, Marie-Thérèse, Marion, Odile, Pascal, Shirley, Thomas, Tina et Valérie, animateurs et encadrants des volets grand public et professionnel.

Enfin, remerciements tous particuliers à Jean Jouzel, Nicole Papineau, Valérie Masson-Delmotte, Stéphane Hallegatte et Nicolas Bériot pour leur confiance et leur soutien. 


\section{Forum international de la Météo et du Climat Les dix meilleures photos du concours "Les phénomènes météorologiques extrêmes "}

Parmi la centaine de photos reçues, le jury a sélectionné les dix meilleures qui ont été exposées sur le parvis de l'hôtel de ville de Paris du 28 mars au $1^{\text {er }}$ avril 2013 dans le cadre du Forum, et que nous avons le plaisir de vous présenter dans La Météorologie.

\section{Les dotations}

$1^{\text {er }}$ prix : un appareil photo Olympus OMD et un abonnement d'un an à Réponses Photo.

$2^{\mathrm{e}}$ prix : des ouvrages Water et Power (éd. teNeues) et un abonnement d'un an à Réponses Photo.

$3^{\mathrm{e}}$ prix : un abonnement d'un an à Réponses Photo.

\section{Les trois photos lauréates}

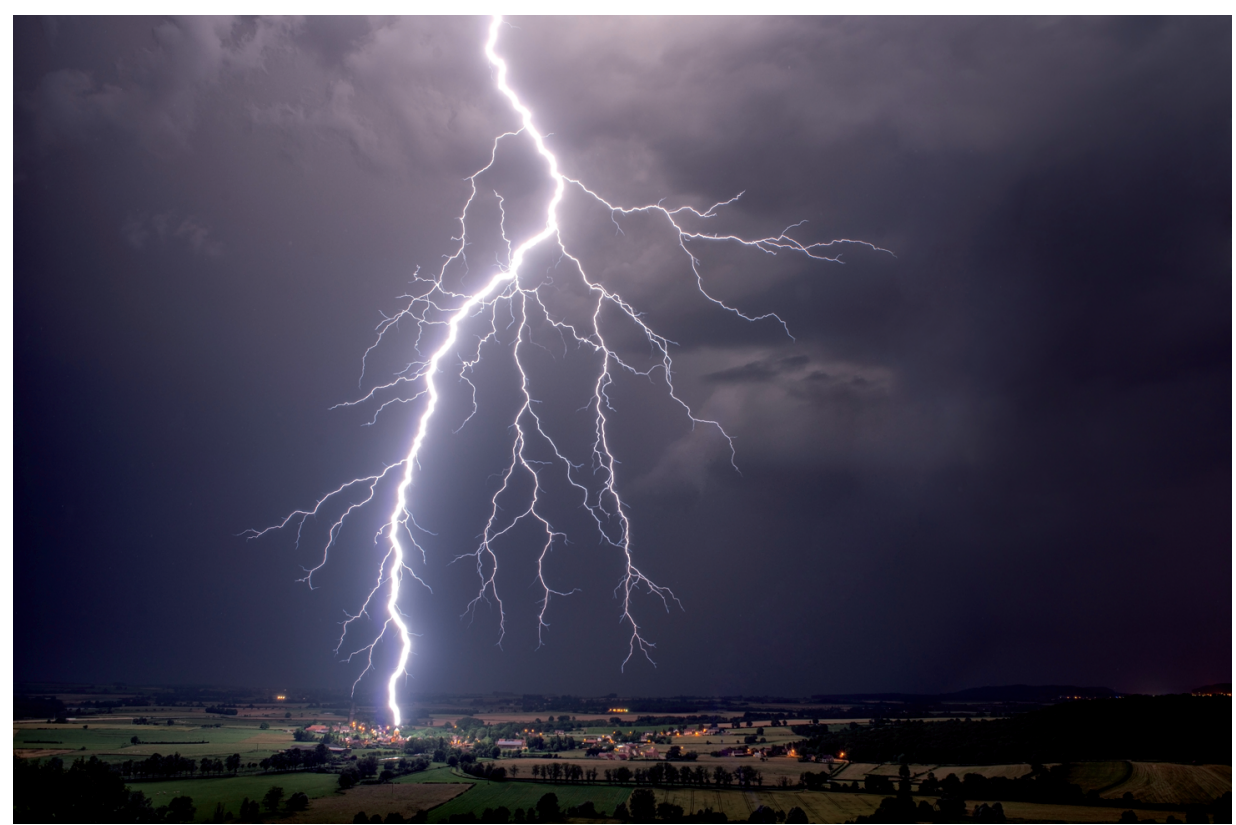

$1^{\text {er }}$ prix - Cyril Leroy

"Coup de foudre sur le clocher de l'église » Vandenesse-en-Auxois (Côte d'Or), 29 juin 2012

\section{$2^{\mathrm{e}}$ prix - Alain Cabanié}

"Tornade dans le sud toulousain "

Goyrans (Haute-Garonne), 29 avril 2012
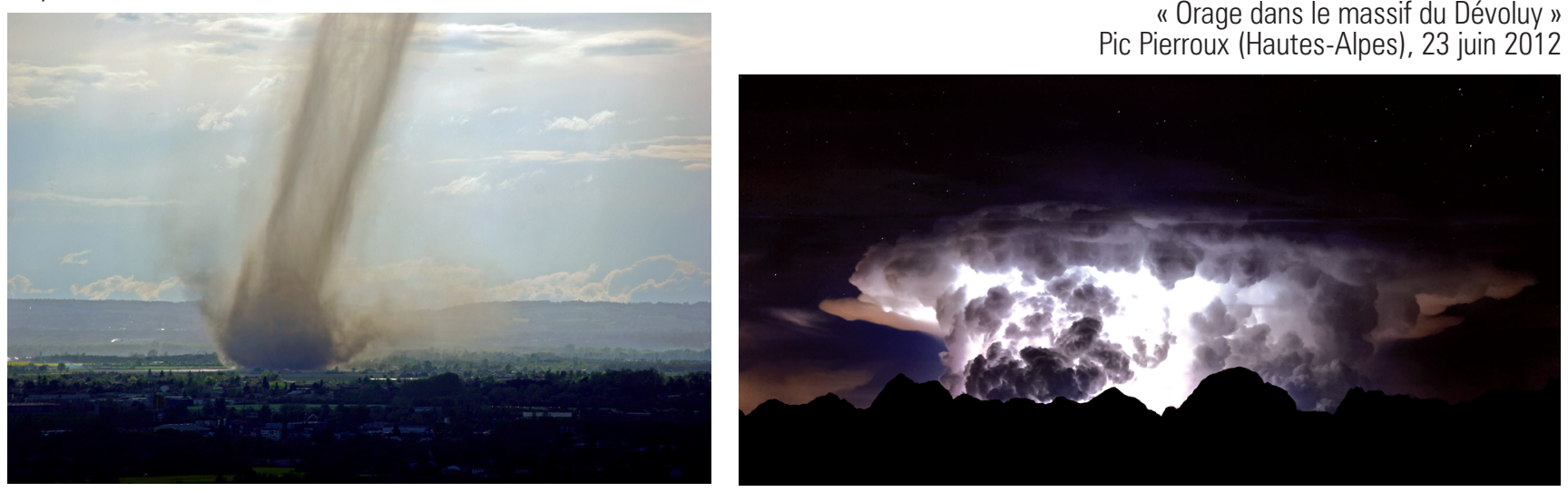


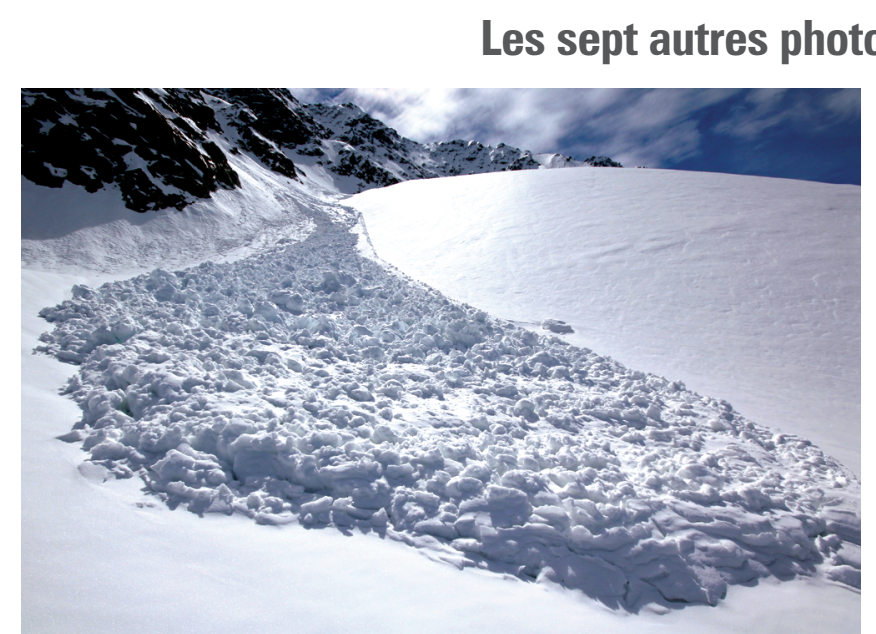

\section{Daniel Goetz}

"Avalanche de neige humide "

Massif de Belledonne (Isère), 5 avril 2011

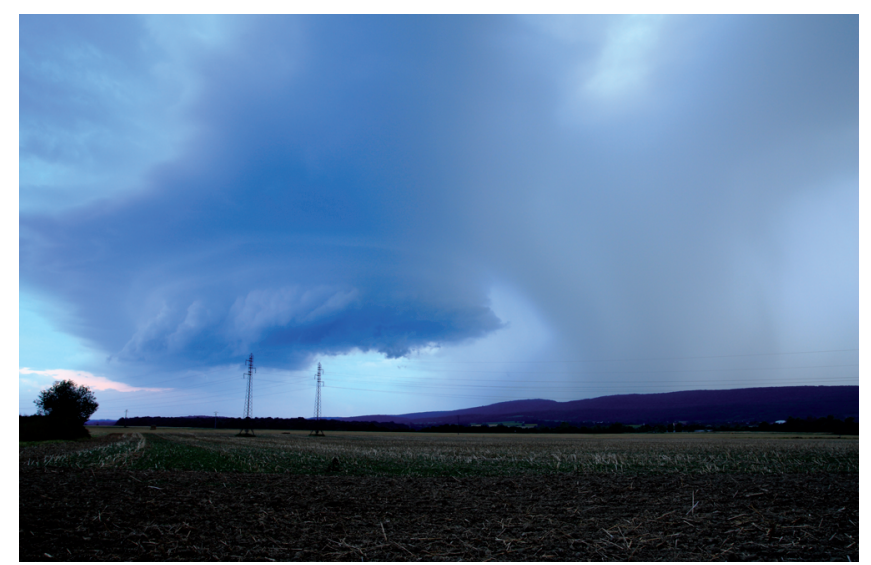

\section{Anthony Xavier}

"Orage supercellulaire »

Région de Villeurbanne (Rhône), 13 juillet 2009

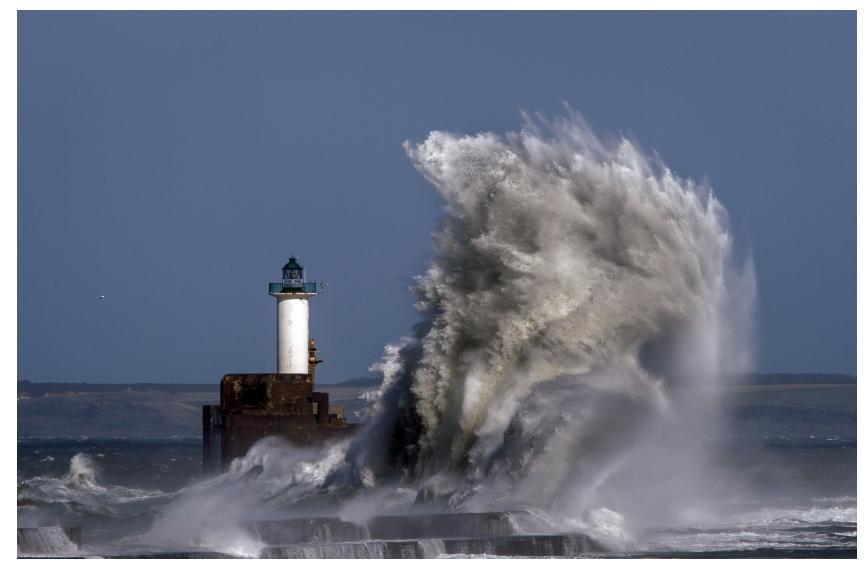

\section{Frédéric Briois}

"L'amiral ». Boulogne-sur-Mer (Pas-de-Calais), novembre 2012

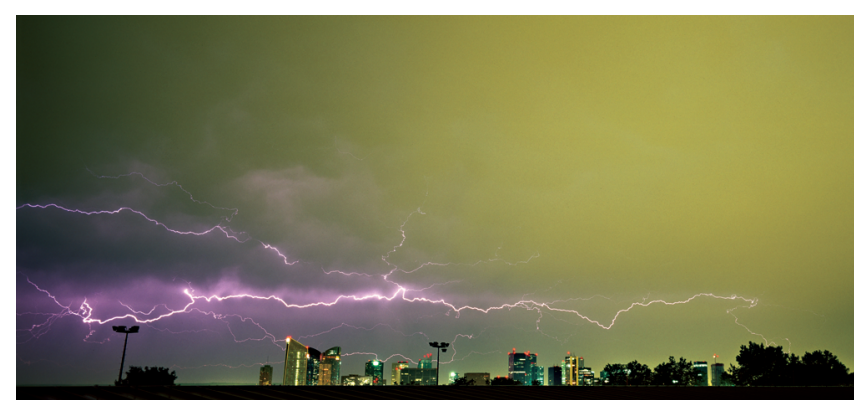

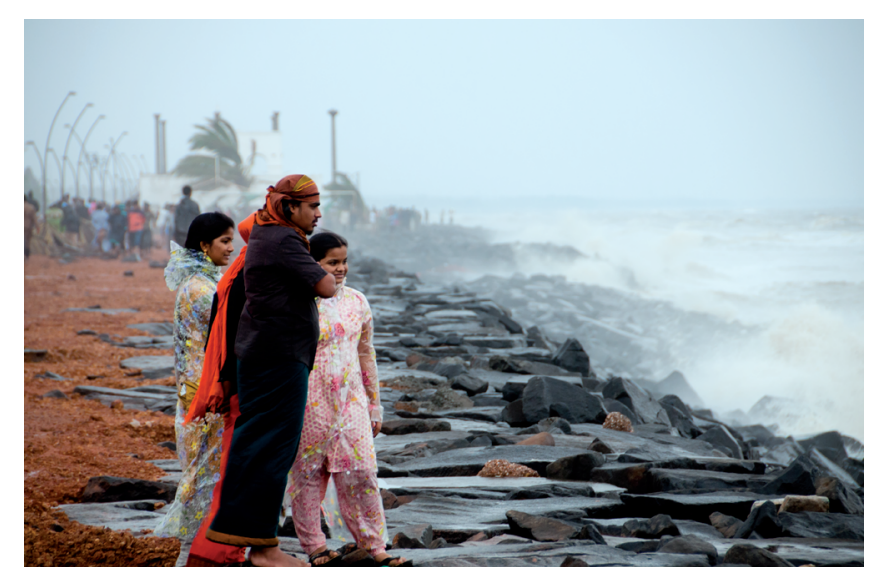

\section{Frédéric Moreau de Bellaing}

"Passage du cyclone Thane "

Côte de Pondichéry (Inde), 30 décembre 2011

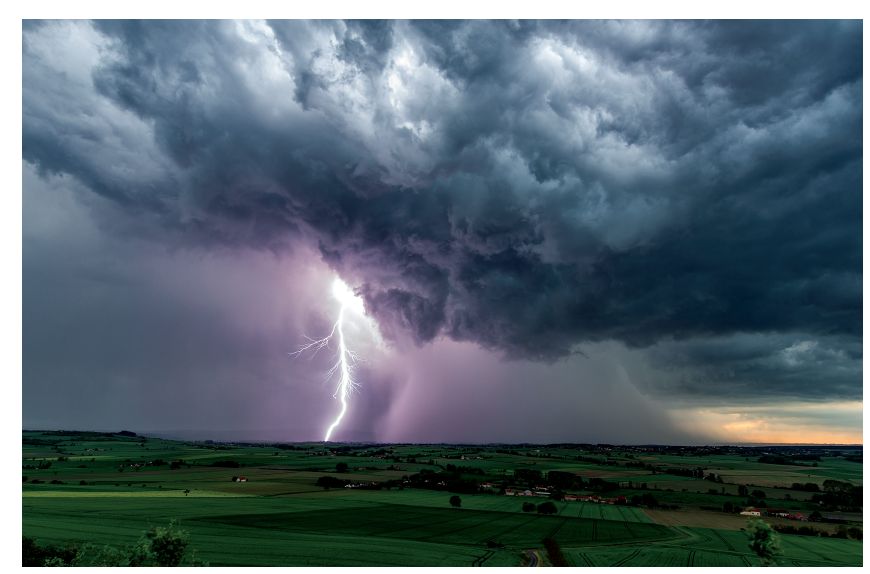

\section{Xavier Delorme}

«Violent orage sur I'Allier ». Étroussat, 26 mai 2012

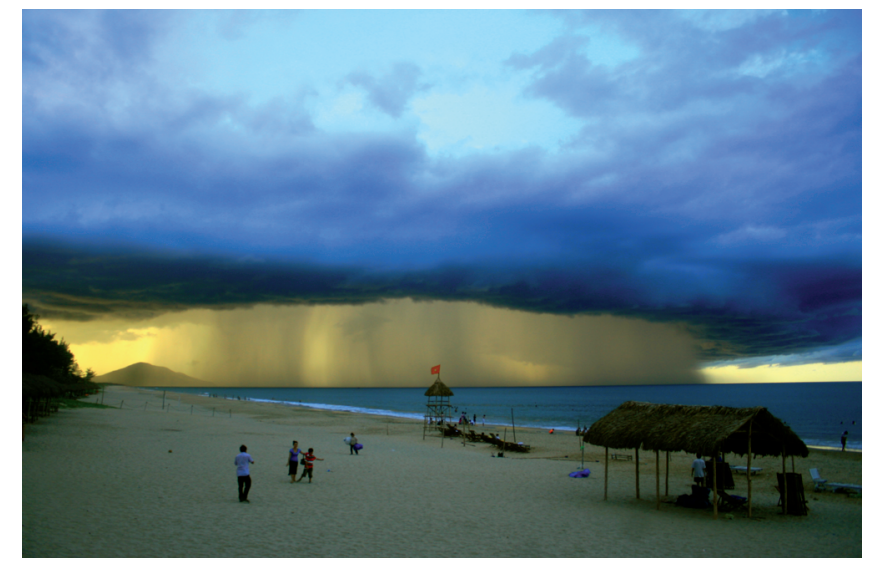

Éric Huynh

«Orage de mousson ». Hoi An (centre du Vietnam), 2009

\section{Michel Launay}

"Multiples éclairs inter-nuageux de fin d'orage »

Suresnes (Hauts-de-Seine), 26 mai 2009 